valle $\langle 0,1\rangle$ on a évidemment $d \psi / d x \geqslant 0$. Posons $\omega=\sup (\xi, \eta)$. En vertu de $(10),(17),(18)$ et $(5)$ nous arons

$$
\Theta(1)=\int_{0}^{1} \int_{0}^{1} f(x s) d s \psi^{\prime}(x) d x \leqslant \int_{\omega}^{1} \int_{0}^{1} f(x s) d s \psi^{\prime}(x) d x<0,
$$

en contradiction avec (11). Ainsi, en supposant pour $t_{0}=1$ l'inégalité $t^{-1} g(t) \leqslant 0$ vérifiée pour tout $t$, nous avons été amenés à une contradiction. Par conséquent la fonction $t^{-1} g(t)$ admet dans l'intérieur de l'intervalle $\langle 0,1\rangle$ un maximum positif. Posons $f^{*}(x)=-f(x)$. La fonction $g^{*}(x)$ $=-g(x)$ qui correspond à la fonction $f^{*}(x)$ admet dans l'intérieur de l'intervalle $\langle 0,1\rangle$ un maximum négatif. Comme nous l'avons montré, la fonction $f^{*}(x)$ est alors identiquement nulle et il en est de même de la fonction $f(x)$.

\title{
TRAVAUX OITESS
}

[1] T. Bonnesen und W. Fenchel, Theorie der konvexen Körper, Berlin 1934

[2] S. Gołab, P 40, Colloquium Mathematicum 1 (1948), p. 240-241.

UNIVERSITE DE ŁÓDŹ

Rę̧u par la Rédaction le 10. 3. 1958

1959

FASC. 1

\section{UNE REMARQUE SUR LA PROPRIETTÉ DE WEIERSTRASS \\ PAR}

\section{H. FAST (WROCEAW)}

Nous disons qu'une fonction réelle $f(x)$, définie sur la droite entière ou dans un intervalle, satisfait à la condition $W\left({ }^{1}\right)(f(x) \epsilon W)$, si l'ensemble des valeurs admises par $f(x)$ dans un intervalle quelconque $\left(x^{\prime}, x^{\prime \prime}\right)$ contient tous les nombres entre $f\left(x^{\prime}\right)$ et $f\left(x^{\prime \prime}\right)$.

Soit $F(x, y)$ une fonction réelle arbitraire définie sur le plan entier. Nous allons démontrer le théorème suivant:

THÉORÈME. Il existe une fonction réelle $u(x)$ définie sur la droize entière telle que la fonction $F\left(x, y_{0}\right)+u(x)$ satisfait à la condition $W$ pour chaque $y_{0}$ fixé.

Démonstration. Définissons deux fonctions $g(x)$ et $h(x)$ de la manière suivante: pour un nombre $x$ dont la partie fractionnelle $x-[x]$ a un développement dyadique de la forme

(1) $\quad 0, a_{0} a_{1} a_{2} \ldots a_{k} 0 \overbrace{11 \ldots 1}^{m} 0 \overbrace{11 \ldots 1}^{n} 0 \overbrace{11 \ldots 1}^{p} \overbrace{11 \ldots 1}^{q} 0 b_{0} 0 b_{1} 0 b_{2} 0 b_{3} 0 \ldots$

$$
\left(a_{i}, b_{i}=0,1 ; q \geqslant 2 ; k, m, n, p \geqslant 0\right)
$$

posons

$$
\begin{aligned}
g(x)-(m-n) & =0, b_{1} b_{3} b_{5} b_{7} \ldots \\
h(x)-(p-q) & =0, b_{0} b_{2} b_{4} b_{6} \ldots
\end{aligned}
$$

Pour les autres valeurs de $x$ posons $g(x)=h(x)=0$. Ainsi, les fonctions $g(x)$ et $h(x)$ sont bien déterminées, puisque, évidemment, le développement (1) détermine d'une manière unique la suite $b_{0}, b_{1}, b_{2}, b_{3}, \ldots$ et les nombres $m, n, p, q$.

LEMME. Pour tout nombre $y$ et pour tout intervalle $\left(x_{1}, x_{2}\right)$ l'ensemble $h\left(g^{-1}(y) \frown\left(x_{1}, x_{2}\right)\right)$ contient tous les nombres réels.

(1) dite propriété de Weierstrass ou propriété de Darboux. 
En effet, des nombres $y$ et $z$ étant donnés arbitrairement, soient

$$
y-[y]=0, b_{1} b_{3} b_{5} \ldots \quad \text { et } \quad z-[z]=0, b_{2} b_{4} b_{6} \ldots
$$

les développements dyadiques (qui peuvent être finis) de leurs parties fractionnelles.

Soient

(3) $\quad 0, a_{0} a_{1} \ldots a_{k} 000 \ldots \quad$ et $0, a_{0} a_{1} \ldots a_{k} 1000 \ldots \quad\left(a_{i}=0,1\right)$

deux nombres de l'intervalle $\left(x_{1}+r, x_{2}+r\right) \frown(0,1)$ oì $r$ est un nombre entier convenable. Désignons par $m, n, p$ et $q$ des entiers positifs pour lesquels

$$
[y]=m-n, \quad[z]=p-q
$$

et par $x$ le nombre dont le développement est de la forme (1), où $a_{i}, b_{i}$, $m, n, p$ et $q$ sont donnés par (2)-(4). Nous avons alors $x-r \in\left(x_{1}, x_{2}\right)$, $g(x)=y, h(z)=z$, d'où là conclusion du lemme.

Définissons la fonction $u(x)$ par la formule suivanțe:

$$
u(x)=h(x)-F(x, g(x)) .
$$

Pour tout $y$ réel nous avons

$$
F(x, y)+u(x)=h(x) \quad \text { pour } \quad x \in g^{-1}(y) .
$$

$\mathrm{Du}$ lemme et de (5) nous déduisons que pour tout $y$ fixé la fonction $F(x, y)+u(x)$ de la variable $x$ prend toutes les valeurs réelles sur chaque intervalle, donc elle satisfait à la condition. $W$, c. q. f. d.

Les deux corollaires suivants résultent de notre théorème, si la fonction $F(x, y)$ est choisie d'une manière convenable.

1. Soit $f(x)$ une fonction arbitraire d'une variable réelle définie sur la droite entière. Posons

$$
F(x, y)=\left\{\begin{array}{ccc}
f(x) & \text { pour } & y \neq 0 \\
0 & \text { pour } & y=0 .
\end{array}\right.
$$

Notre théorème entraine dans ce cas que $f(x)+u(x) \epsilon W$ et en posant $y=0$, que $u(x) \epsilon W$, d'où $-u(x) \epsilon W$. Ainsi, toute fonation $f(x)$ est la sommo de deux fonctions $(f(x)+u(x)$ et $-u(x))$ satisfaisant $\dot{a}$ la condition $W$ Ce théorème, publié sans démonstration par A. Lindenbaum, a été aussi démontré par Sierpiński $\left({ }^{2}\right)$.

(2) W. Sierpiński, Sur une propriété des fonctions réelles quelconques, Le Matematiche 8 (1953), p. $1-6$.
2. Soit encore $f(x)$ une fonction réelle arbitraire définie sur la droite entière. Soit maintenant

$$
F(x, y)=\left\{\begin{array}{l}
(n-1) \cdot f(x) \text { pour } y=n(n=1,2, \ldots), \\
\text { arbitraire si } y \text { n'est pas un entier positif. }
\end{array}\right.
$$

En vertu du théorème (p. 75) il existe une fonction $u(x)$ telle que $(n-1) \cdot f(x)+u(x) \epsilon W(n=1,2, \ldots)$, d'où l'on déduit, en divisant par $n$, que

$$
f_{n}(x)=\left(1-\frac{1}{n}\right) \cdot f(x)+\frac{1}{n} \cdot u(x) \in W \quad(n=1,2, \ldots) .
$$

En faisant tendre $n$ vers l'infini nous avons $f(x)=\lim f_{n}(x)$, c'est-à-dire: une fonction réelle arbitraire est la limite d'une suite de fonctions ayant la propriété $W$.

C'est la réponse affirmative à une question que m'a posée S. Hart$\operatorname{man}\left({ }^{3}\right)$.

( $\left.{ }^{8}\right)$ Sierpiński a signalé cette réponse sans démonstration (voir $\left.{ }^{(2)}\right)$.

Reçu par la Rédaction le 1. 9. 1958 